Corrigendum

\title{
Corrigendum to "Clinicopathological Characteristics and Prognosis of Papillary Thyroid Carcinoma in Naturally Menopausal Women with Various Durations of Premenarche, Reproductive Periods, and Postmenopausal Stages"
}

\author{
Xu-Hang Zhu, ${ }^{1,2}$ Bin Yu, ${ }^{1,2}$ Yu-qing Huang, ${ }^{1,2}$ Jing-nan Zhou, ${ }^{3}$ and Ming-Hua Ge ${ }^{2}$ \\ ${ }^{1}$ Second Clinical Medical College, Zhejiang Chinese Medical University, Binjiang District, Hangzhou 310022, China \\ ${ }^{2}$ Department of Head and Neck Surgery, Zhejiang Province Cancer Hospital, Gongshu District, Hangzhou 310022, China \\ ${ }^{3}$ Department of Equipment, Zhejiang Province Cancer Hospital, Gongshu District, Hangzhou 310022, China \\ Correspondence should be addressed to Ming-Hua Ge; gemh@zjcc.org.cn \\ Received 8 March 2018; Accepted 20 March 2018; Published 2 May 2018 \\ Copyright (c) $2018 \mathrm{Xu}$-Hang Zhu et al. This is an open access article distributed under the Creative Commons Attribution License, \\ which permits unrestricted use, distribution, and reproduction in any medium, provided the original work is properly cited.
}

In the article titled "Clinicopathological Characteristics and Prognosis of Papillary Thyroid Carcinoma in Naturally Menopausal Women with Various Durations of Premenarche, Reproductive Periods, and Postmenopausal Stages" [1], the name of the first author was given incorrectly as Xuhang Zhu. The author's name should have been written as $\mathrm{Xu}$-Hang $\mathrm{Zhu}$. The revised authors' list is shown above.

\section{References}

[1] X.-h. Zhu, Y. Bin, Y.-q. Huang, J.-n. Zhou, and M.-H. Ge, "Clinicopathological characteristics and prognosis of papillary thyroid carcinoma in naturally menopausal women with various durations of premenarche, reproductive periods, and postmenopausal stages," International Journal of Endocrinology, vol. 2017, Article ID 5702716, 11 pages, 2017. 


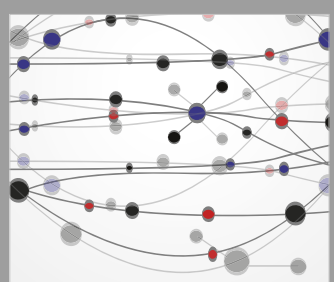

The Scientific World Journal
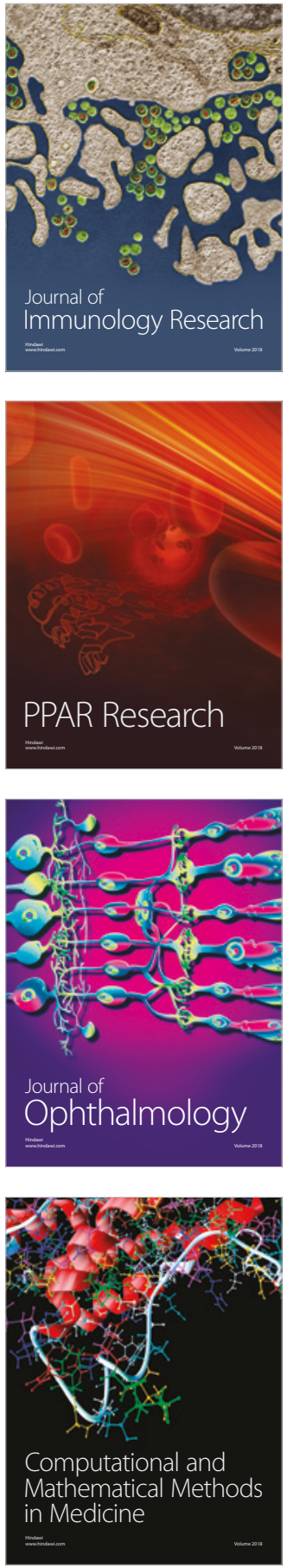

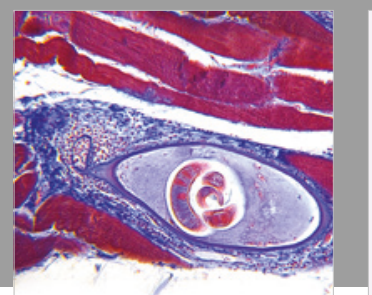

Gastroenterology Research and Practice

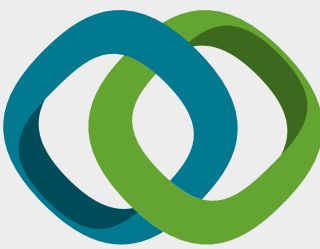

\section{Hindawi}

Submit your manuscripts at

www.hindawi.com
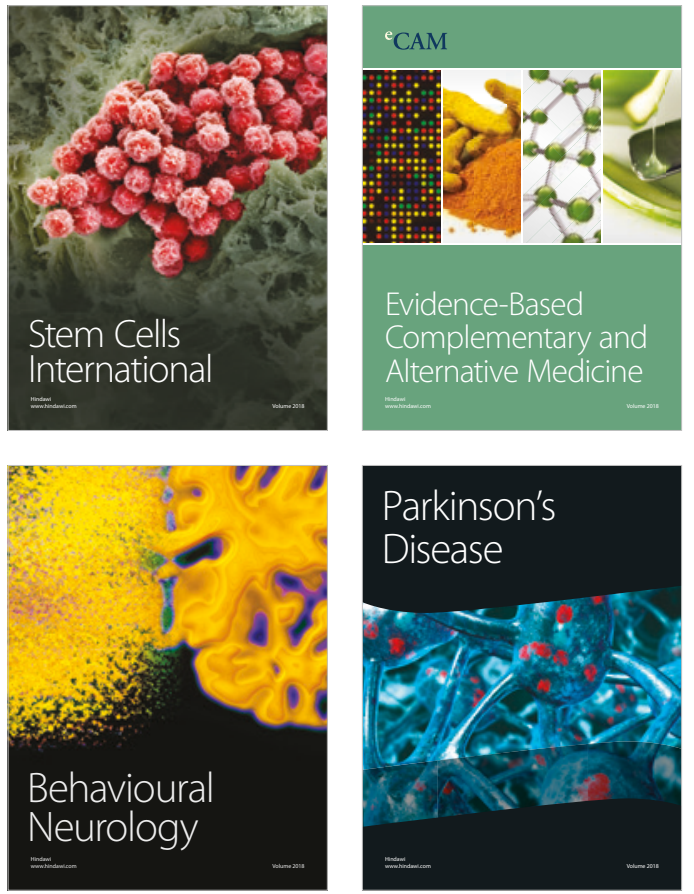

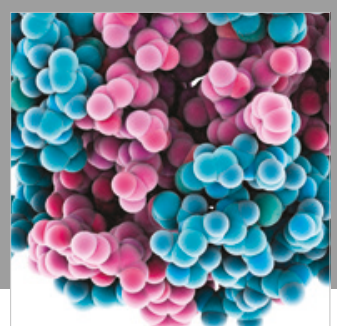

ournal of

Diabetes Research

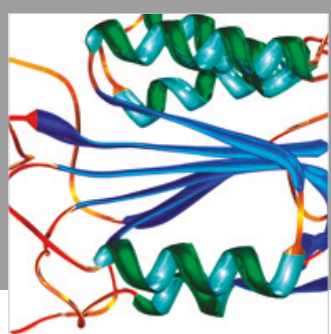

Disease Markers
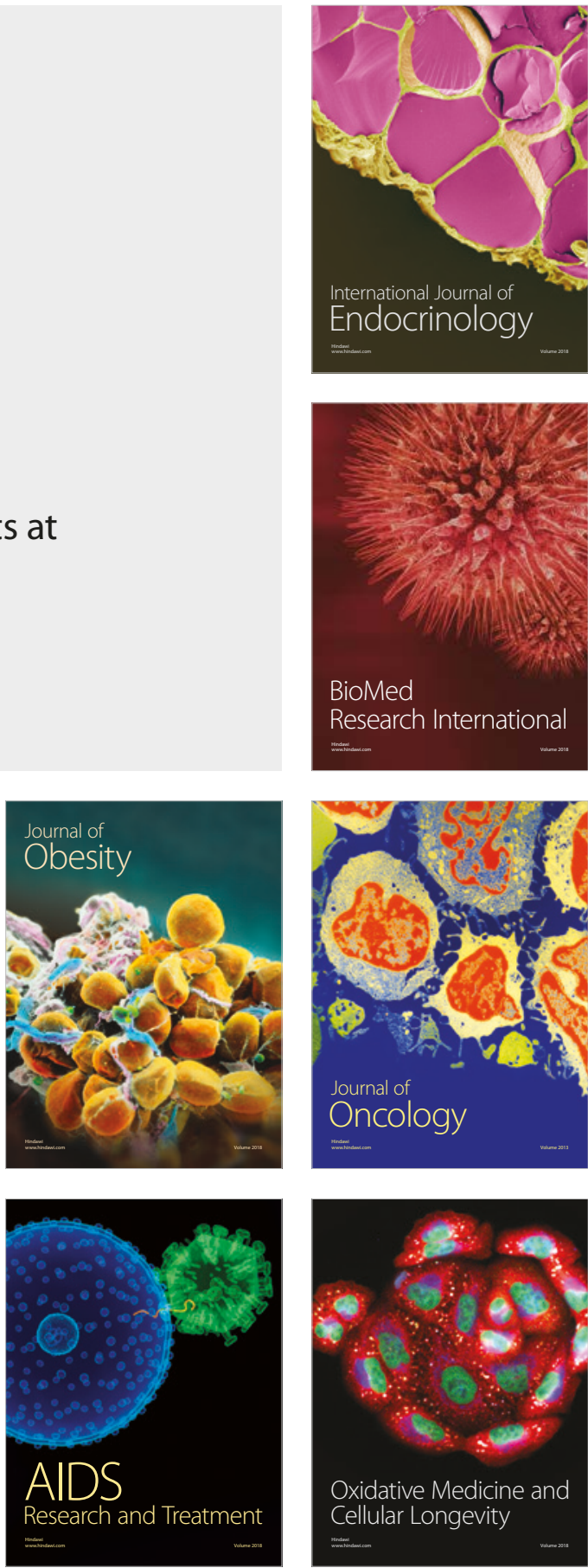